\title{
An improved CE/SE scheme and its application to dilute gas-particle flows
}

\author{
Gang Wang ${ }^{\mathrm{a}, *}$, Huiyu Zhu ${ }^{\mathrm{a}}$, Quanhua Sun ${ }^{\mathrm{a}}$, Deliang Zhang ${ }^{\mathrm{a}}$, Kaixin Liu ${ }^{\mathrm{b}}$ \\ a LHD, Institute of Mechanics, Chinese Academy of Sciences, Beijing, China \\ ${ }^{\mathrm{b}}$ LTCS and College of Engineering, Peking University, Beijing, China
}

\section{A R T I C L E I N F O}

\section{Article history:}

Received 15 October 2010

Received in revised form 2 March 2011

Accepted 7 April 2011

Available online 13 April 2011

\section{Keywords:}

CE/SE scheme

Numerical simulations

Dilute gas-particle flows

\begin{abstract}
A B S T R A C T
An improved space-time Conservation Element and Solution Element (CE/SE) scheme is constructed by proposing a new structure of solution elements and conservation elements based on the rectangular mesh. Furthermore, the improved CE/SE scheme was applied to dilute gas-particle two-phase flows. A two-fluid model and two corresponding chemical reaction models, i.e., two-step reaction model and detailed chemical reaction model, were used to describe the physical and chemical characteristics in the two-phase flows. Shock wave reflection in gas, shock wave diffraction in air-sand mixture, explosive synthesis of $\mathrm{TiO}_{2}$ nanoparticle and air-fuel two-phase detonation were simulated by the improved CE/SE scheme and appropriate physical and chemical models. All the numerical results were compared and discussed carefully. The results show that the improved CE/SE scheme is clear in physical concept, easy to be implemented and high accurate for the above-mentioned problems. Thus, the improved CE/SE scheme can be applied to gas-particle flows widely.
\end{abstract}

(c) 2011 Elsevier B.V. All rights reserved.

\section{Introduction}

Numerical simulations have become popular and are playing more and more important roles in the fluid dynamics research. But the numerical simulations of gas-particle two-phase flows are very difficult due to the complex physical and chemical phenomena. Numerical simulations of gas-particle flows need not only accurate numerical schemes but also appropriate physical and chemical models. Recently, the numerical research of gas-particle flow is mainly focus on method research [1] and engineering application [2]. Method research includes numerical schemes, single particle models, treatment of particle phase, turbulence models, etc. Engineering application involves many areas, such as aerospace, industrial production and military defense, etc.

The space-time Conservation Element and Solution Element (CE/SE) method proposed originally by Chang $[3,4]$ is a new high resolution CFD (Computational Fluid Dynamics) method for conservation laws. It substantially differs from other traditional numerical methods by possessing many features, e.g., unified treatment of space and time, new shock-capturing strategy, satisfaction of both local and global flux conservations in space and time by introducing conservation elements (CEs) and solution elements (SEs). Simplicity, generality and accuracy are the three main advantages of the CE/SE method [3]. The CE/SE method has gained great successes in simulations of inviscid supersonic flows [5,6], steady viscous flows [7], MHD (Magneto-Hydro-Dynamic) flows [8,9], multi-material elastic-plastic flows [10], chemically reacting flows [11-13], etc. However, the original CE/SE method also has some disadvantages, such as complicated mesh structure for two-dimensional situation, and the shortage of high-accuracy schemes. Zhang et al. [6] have proposed quadrilateral and hexahedral meshes for two- and three-dimensional CE/SE schemes and obtained satisfying results. Liu and Wang [14] have proposed one-dimensional high-accuracy CE/SE schemes and analyzed the stability of the second-order CE/SE scheme. Wang et al. [15,16] proposed two-dimensional high-accuracy CE/SE schemes based on the rectangular mesh.

The aim of the present work is to construct a simple and accurate two-dimensional CE/SE scheme for dilute gas-particle flows. A new structure of CEs and SEs based on rectangular mesh is proposed and corresponding numerical schemes are constructed. To verify the accuracy of the improved $\mathrm{CE} / \mathrm{SE}$ scheme, shock wave reflection in gas, shock wave diffraction in air-sand mixture, explosive synthesis of $\mathrm{TiO}_{2}$ nanoparticle and air-fuel two-phase detonation were simulated aiding with a two-fluid model and two chemical reaction models. All numerical results were compared and discussed in detail.

\footnotetext{
* Corresponding author. Tel.: +861381158 0863.

E-mail address: wanggang@imech.ac.cn (G. Wang).
} 


\section{Numerical models}

The number of particles in a two-phase flow may be very huge. So using Lagrangian method to trace all particles needs a lot of computational source. And for some problems, such as the explosive synthesis of $\mathrm{TiO}_{2}$ nanoparticle, it is impossible to trace all particles due to the large numbers of particles, which is approximately equals to $10^{18} / \mathrm{m}^{3}$ in our simulations. In this paper, a two-fluid model is introduced for treating two-phase flows. The two-fluid model considers the particle phase as continuous medium, so continuous medium mechanics can be used to describe the flow of particle phase. It is assumed that the characters of considered dilute gas-particle systems in this paper as follows: the flow is unsteady; the initial temperature of the gas and the particle is the same; the radii of particles are uniform; the phase of particles is considered as a continuous medium; the shape of particles always keeps to be spherical even in process of growth, separation, evaporation, etc.; the temperature distribution in particles is uniform; the interactions between particles are ignored; if chemical reaction occurs, the chemical energy is absorbed only by gas, which is considered as ideal.

Under the above assumptions the flow in gas-particle mixture is governed by Euler's equations

$$
\frac{\partial \mathbf{Q}}{\partial t}+\frac{\partial \mathbf{E}}{\partial x}+\frac{\partial \mathbf{F}}{\partial y}=\mathbf{S}
$$

In Eq. (1), $\mathbf{Q}$ is the vector of conserved variables, $\mathbf{E}$ and $\mathbf{F}$ are conservation flux vectors in the $x$ - and $y$-directions, $\mathbf{S}$ is the source term vector.

If there is no chemical reaction and phase change, for example, air-sand mixture, the expressions of $\mathbf{Q} \mathbf{E}, \mathbf{F}, \mathbf{S}$ are as follows

$$
\mathbf{Q}=\left(\begin{array}{l}
\rho_{g} \\
\rho_{g} u_{g} \\
\rho_{g} v_{g} \\
E_{g} \\
\rho_{p} \\
\rho_{p} u_{p} \\
\rho_{p} v_{p} \\
N
\end{array}\right), \quad \mathbf{E}=\left(\begin{array}{l}
\rho_{g} u_{g} \\
\rho_{g} u_{g}^{2}+\varphi_{g} p \\
\rho_{g} u_{g} v_{g} \\
\left(E_{g}+\varphi_{g} p\right) u_{g} \\
\rho_{p} u_{p} \\
\rho_{p} u_{p}^{2} \\
\rho_{p} u_{p} v_{p} \\
N u_{p}
\end{array}\right), \quad \mathbf{F}=\left(\begin{array}{l}
\rho_{g} v_{g} \\
\rho_{g} u_{g} v_{g} \\
\rho_{g} v_{g}^{2}+\varphi_{g} p \\
\left(E_{g}+\varphi_{g} p\right) v_{g} \\
\rho_{p} v_{p} \\
\rho_{p} u_{p} v_{p} \\
\rho_{p} v_{p}^{2} \\
N v_{p}
\end{array}\right), \quad \mathbf{S}=\left(\begin{array}{l}
0 \\
-F_{x} \\
-F_{y} \\
-u_{p} F_{x}-v_{p} F_{y} \\
0 \\
F_{x} \\
F_{y} \\
0
\end{array}\right),
$$

where $\rho_{g}$ is the mass density of gas phase, $u_{g}$ and $v_{g}$ are the velocity components of gas phase, $p$ is the pressure, $E_{g}$ is the total energy density of gas phase, $\varphi_{\mathrm{g}}$ is the volume percent of gas phase, $\rho_{p}$ is the mass density of particle phase, $u_{p}$ and $v_{p}$ are the velocity components of particle phase, $N$ is the particle number per unit volume, $F_{X}$ and $F_{y}$ are the force components acting on particles.

The volume percent of gas phase is defined as

$$
\varphi_{g}=1-\varphi_{p}=1-\frac{\rho_{p}}{\rho_{\text {matter }}},
$$

where $\varphi_{p}$ is the volume percent of particle phase; $\rho_{\text {matter }}$ is the density of compact material, such as solid sand $\rho_{\text {sand }}$, liquid fuel $\rho_{\text {fuel }}$ and solid $\mathrm{TiO}_{2} \rho_{\text {titania }}$ in this work. According to our assumptions, $\rho_{p}$ and $\rho_{\text {matter }}$ have the relation:

$$
\rho_{p}=N \times \frac{4}{3} \pi r^{3} \rho_{\text {matter }},
$$

where $r$ is the radius of particles.

The total energy density of gas phase, $E$, is defined as

$$
E_{g}=\frac{\varphi_{g} p}{\gamma-1}+\frac{\rho_{g}}{2}\left(u_{g}^{2}+v_{g}^{2}\right)
$$

where $\gamma$ is the specific heat ratio.

The force components on particles are expressed as $[17,18]$

$$
\left\{\begin{array}{l}
F_{x}=0.5 \pi r^{2} C_{D} \rho_{g}\left|V_{g}-V_{p}\right|\left(u_{g}-u_{p}\right) N \\
F_{y}=0.5 \pi r^{2} C_{D} \rho_{g}\left|V_{g}-V_{p}\right|\left(v_{g}-v_{p}\right) N
\end{array}\right.
$$

with

$$
\begin{aligned}
& \left|V_{g}-V_{p}\right|=\left[\left(u_{g}-u_{p}\right)^{2}+\left(v_{g}-v_{p}\right)^{2}\right]^{1 / 2}, \\
& C_{D}= \begin{cases}27 R e^{-0.84}, & R e<80, \\
0.27 \operatorname{Re}^{0.21}, & 80 \leqslant \operatorname{Re}<10^{4}, \\
2, & \operatorname{Re} \geqslant 10^{4},\end{cases} \\
& \operatorname{Re}=\frac{2 \rho_{p} r\left|V_{g}-V_{p}\right|}{\mu_{g}},
\end{aligned}
$$

where $\mu_{\mathrm{g}}$ is the viscosity coefficient of gas phase, $C_{D}$ is the drag coefficient, Re means Reynolds number.

A two-step reaction model is adopted for the detonation synthesis simulations. The two-step reaction model simplifies the complicated chemical reactions to an induction reaction and an exothermic reaction [19]. The progress parameters $\alpha$ and $\beta$, for the induction reaction 
and the exothermic reaction, respectively, are both equal to zero first, followed by increase of $\alpha$ to unit, then $\beta$ increases until the equilibrium state is reached. The rates of $\alpha$ and $\beta, \omega_{\alpha}$ and $\omega_{\beta}$, are given as follows:

$$
\begin{array}{ll}
\omega_{\alpha}=\frac{d \alpha}{d t}=k_{\alpha} \rho_{g} \exp \left(-\frac{E_{a}}{R T}\right), \\
\omega_{\beta}= \begin{cases}0, & \alpha<1, \\
k_{\beta} \varphi_{g}^{2} p^{2}(1-\beta)^{2} \exp \left(-\frac{E_{\beta}}{R T}\right), & \alpha \geqslant 1,\end{cases}
\end{array}
$$

where $k_{\alpha}$ and $k_{\beta}$ are the constants of reaction rate, $E_{\alpha}$ and $E_{\beta}$ are the activation energies, $R$ is the gas constant, $T$ is the temperature.

For the two-step reaction model, the governing equation (1) should consider the conservation of the progress parameters $\alpha$ and $\beta$. So the expressions of $\mathbf{Q} \mathbf{E}, \mathbf{F}, \mathbf{S}$ are as follows:

$$
\mathbf{Q}=\left(\begin{array}{l}
\rho_{g} \\
\rho_{g} u_{g} \\
\rho_{g} v_{g} \\
E_{g} \\
\rho_{g} \alpha \\
\rho_{g} \beta \\
\rho_{p} \\
\rho_{p} u_{p} \\
\rho_{p} v_{p} \\
N
\end{array}\right), \quad \mathbf{E}=\left(\begin{array}{l}
\rho_{g} u_{g} \\
\rho_{g} u_{g}^{2}+\varphi_{g} p \\
\rho_{g} u_{g} v_{g} \\
\left(E_{g}+\varphi_{g} p\right) u_{g} \\
\rho_{g} \alpha u_{g} \\
\rho_{g} \beta u_{g} \\
\rho_{p} u_{p} \\
\rho_{p} u_{p}^{2} \\
\rho_{p} u_{p} v_{p} \\
N u_{p}
\end{array}\right), \quad \mathbf{F}=\left(\begin{array}{l}
\rho_{g} v_{g} \\
\rho_{g} u_{g} v_{g} \\
\rho_{g} v_{g}^{2}+\varphi_{g} p \\
\left(E_{g}+\varphi_{g} p\right) v_{g} \\
\rho_{g} \alpha v_{g} \\
\rho_{g} \beta v_{g} \\
\rho_{p} v_{p} \\
\rho_{p} u_{p} v_{p} \\
\rho_{p} v_{p}^{2} \\
N v_{p}
\end{array}\right), \quad \mathbf{S}=\left(\begin{array}{l}
-I_{p} \\
-F_{x}-u_{p} I_{p} \\
-F_{y}-v_{p} I_{p} \\
-u_{p} F_{x}-v_{p} F_{y}+0.5\left(u_{p}^{2}+v_{p}^{2}\right) I_{p} \\
\rho_{g} \omega_{\alpha} \\
\rho_{g} \omega_{\beta} \\
I_{p} \\
F_{x}+u_{p} I_{p} \\
F_{y}+v_{p} I_{p} \\
0
\end{array}\right),
$$

where $I_{p}$ is the density variation during phase change. Due to the chemical energy release, the total energy density of gas phase, $E_{g}$, is defined as

$$
E_{g}=\frac{\varphi_{g} p}{\gamma-1}+\frac{\rho_{g}\left(u_{g}^{2}+v_{g}^{2}\right)}{2}+\rho_{g} \beta Q
$$

where $Q$ is the reaction energy. In the simulation of detonation synthesis, $I_{p}$ is the rate of particle growth, which is coupled with the chemical reaction model [20].

A detailed chemical reaction model is used for simulating gas-droplet two-phase detonations in this paper. The detailed chemical reaction model is extensively used to describe the transformation of reactants into products at the molecular level through a large number of elementary steps. Concentrations of reactants, intermediates and products can be computed by integrating the sets of differential equations describing the rates of formation and destruction of each species. The production rate of the $i$ th chemical specie, $\omega_{i}$, is

$$
\omega_{i}=W_{i} \sum_{k=1}^{n r}\left(v_{k i}^{\prime \prime}-v_{k i}^{\prime}\right) R P_{k},
$$

where $W_{i}$ is the molecular weight of the $i$ th specie, $n r$ is the number of elementary step, $v_{k i}^{\prime}$ and $v_{k i}^{\prime \prime}$ are the stoichiometric coefficients, $R P_{k}$ is the rate of process variable of the $k$ th element reaction whose detailed computing formula can be found in Ref. [21].

For the detailed chemical reaction model, the governing equation (1) should consider the conservations of every species. So the expressions of $\mathbf{Q}, \mathbf{E}, \mathbf{F}, \mathbf{S}$ are as follows:

$$
\mathbf{Q}=\left(\begin{array}{l}
\rho_{g, 1} \\
\rho_{g, 2} \\
\vdots \\
\rho_{g, n s} \\
\rho_{g} u_{g} \\
\rho_{g} u_{g} \\
E_{g} \\
\rho_{p} \\
\rho_{p} u_{p} \\
\rho_{p} v_{p} \\
N
\end{array}\right), \quad \mathbf{E}=\left(\begin{array}{l}
\rho_{g, 1} u_{g} \\
\rho_{g, 2} u_{g} \\
\vdots \\
\rho_{g, n s} u_{g} \\
\rho_{g} u_{g}^{2}+\varphi_{g} p \\
\rho_{g} u_{g} v_{g} \\
\left(E_{g}+\varphi_{g} p\right) u_{g} \\
\rho_{p} u_{p} \\
\rho_{p} u_{p}^{2} \\
\rho_{p} u_{p} v_{p} \\
N u_{p}
\end{array}\right), \quad \mathbf{F}=\left(\begin{array}{l}
\rho_{g, 1} v_{g} \\
\rho_{g, 2} v_{g} \\
\vdots \\
\rho_{g, n s} v_{g} \\
\rho_{g} u_{g} v_{g} \\
\rho_{g} v^{2}+\varphi_{g} p \\
\left(E_{g}+\varphi_{g} p\right) v_{g} \\
\rho_{p} v_{p} \\
\rho_{p} u_{p} v_{p} \\
\rho_{p} v_{p}^{2} \\
N v_{p}
\end{array}\right), \quad \mathbf{S}=\left(\begin{array}{l}
I_{p}+\omega_{1} \\
\omega_{2} \\
\vdots \\
\omega_{n s} \\
-F_{x}+u_{p} I_{p} \\
-F_{y}+v_{p} I_{p} \\
-u_{p} F_{x}-v_{p} F_{y}+0.5\left(u_{p}^{2}+v_{p}^{2}\right) I_{p} \\
-I_{p} \\
F_{x}-u_{p} I_{p} \\
F_{y}-v_{p} I_{p} \\
0
\end{array}\right),
$$

where $\rho_{g, i}$ ( $i$ from 1 to $n s$ ) is the density of the $i$ th specie (especially, $\rho_{g, 1}$ the density of fuel gas), $n s$ is the number of considered specie. For the detailed chemical reaction model, the total energy density of gas phase, $E_{g}$, is defined as

$$
E_{g}=\rho_{g} h-\varphi_{g} p+\frac{\rho_{g}}{2}\left(u_{g}^{2}+v_{g}^{2}\right)
$$

where $h$ is the enthalpy calculated by the thermochemical relation $h=h\left(\rho_{i}, T\right)$ [22]. 


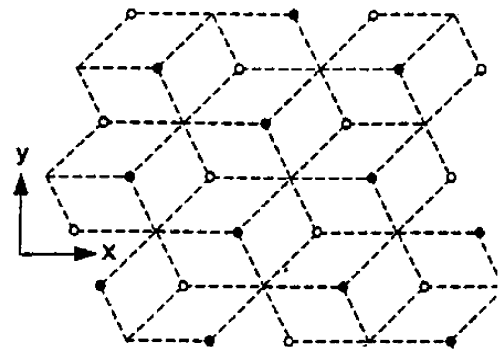

(a) Mesh points projection on the $x-y$ plane

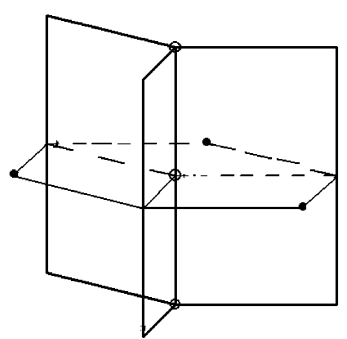

(b) SE

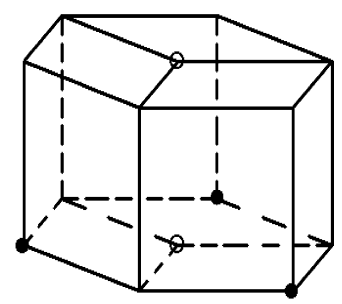

(c) CEs

Fig. 1. Space-time geometrical configuration of the original two-dimensional CE/SE scheme (by Chang).

In the gas-droplet two-phase detonations, the evaporation and separation of fuel droplets should be considered. The density variation, $I_{p}$, can be obtained as $[17,18]$

$$
I_{P}=12 r N \frac{\lambda N u\left(T-T_{p}\right)}{L}+4 \pi r^{3 / 2} \rho_{\text {fuel }} N\left(\frac{\rho_{g} \mu_{g}}{\rho_{\text {fuel }} \mu_{p}}\right)^{1 / 6}\left(\frac{\mu_{p}}{\rho_{\text {fuel }}}\right)^{1 / 2}\left|V_{g}-V_{p}\right|^{1 / 2},
$$

with

$$
N u=2+0.6 \operatorname{Re}^{1 / 2} \operatorname{Pr}^{1 / 3}
$$

where $\lambda$ is the heat conduction coefficient of gas, $T_{p}$ is the temperature of fuel droplets, $L$ is the heat of evaporation of fuel droplets, $\rho_{f u e l}$ is the density of fuel droplets, $\mu_{p}$ is the viscosity coefficient of droplet phase, $N u$ means Nusselt number and $P r$ means Prandtl number. The first term of Eq. (17) describes the evaporation of fuel drops, and the second term describes the shattering of fuel drops.

In conclusion, if the two-fluid model is adopted, the governing equation of dilute gas-particle flows can be express as Eq. (1), thought the three dilute two-phase problems, i.e., shock wave diffraction in air-sand mixture, explosive synthesis of $\mathrm{TiO}_{2}$ nanoparticle and air-fuel two-phase detonation, need different physical and chemical models. So the improved CE/SE schemes will be constructed based on Eq. (1).

\section{The improved CE/SE scheme}

\subsection{Numerical scheme}

The construction of a CE/SE scheme includes the following steps: changing governing equations from differential form to integral form, dividing space-time region as CEs and SEs, approximating flux vectors in SEs by Taylor expansions, obtaining relation equations between the space derivatives and the time derivatives, and finally integrating the governing equations on CEs. So, different definitions of SEs and CEs may construct different numerical CE/SE schemes. Fig. 1 shows the space-time geometrical configuration of Chang's two-dimensional $\mathrm{CE} / \mathrm{SE}$ scheme [4]. Due to the obvious disadvantage that directions of space mesh are neither perpendicular each other nor coincident with global Cartesian coordinate, this special designs of SEs and CEs are neither convenient to construct numerical schemes nor suitable for three-dimensional extension.

We design a new type of SE and CE on the general hexahedrons mesh, which is different from that of the Chang's [23] original CE/SE method (see Fig. 1). The construction of the new numerical scheme is simpler than Chang's and it is easy to be extended to threedimensional problems. A CE/SE scheme corresponding to the new definition of SE and CE would be easy to construct and direct to extend to three-dimensional situation.

The space-time region is divided into the unified mesh points. For the two-dimensional scheme, let $(j, k, n)$ denote a set of space-time mesh points, where $n=0, \pm 1 / 2, \pm 1, \pm 3 / 2, \ldots$ for time, $j=0, \pm 1 / 2, \pm 1, \pm 3 / 2, \ldots$ for $x$ and $k=0, \pm 1 / 2, \pm 1, \pm 3 / 2, \ldots$ for $y$. Fig. 2 (a) shows the projection of mesh points on the $x-y$ plane, in which the interval between the mesh points $\bullet$ and $\circ$ is $\Delta t / 2$ in the time direction or $1 / 2$ in the mesh number $n$. An SE (see Fig. 2(b)) is defined as the vicinity of a mesh point and the whole space-time region is divided into non-overlapping CEs. Assume that the physical variables in every SE are approximated by the Taylor's expansions at the mesh point associated with the SE, and the conservation equations (1) are satisfied in every CE (a cuboid volume related to the SE, see Fig. 2(c)).

Let $x_{1}=x, x_{2}=y, x_{3}=t$ be considered as the coordinates of a Euclidean space $E_{3}$. The conventional integral form of Eq. (1) is

$$
\int_{V} \nabla \cdot \mathbf{H}_{m} d v=\int_{V} S_{m} d v
$$

where $\nabla=(\partial / \partial x, \partial / \partial y, \partial / \partial t), \mathbf{H}_{m}=\left(E_{m}, F_{m}, Q_{m}\right)$ is the space-time flux vector, where $E_{m}, F_{m}$ and $Q_{m}$ are the components of the vector $\mathbf{E}, \mathbf{F}$ and $\mathbf{Q}$ respectively; $S_{m}$ are the components of the source term vector.

By using the Gauss' divergence theorem, Eq. (19) can be rewritten in the form of

$$
\oint_{S(V)} \mathbf{H}_{m} \cdot d \mathbf{s}=\int_{V} S_{m} d v
$$

where $S(V)$ is the boundary of the space-time region $V$ in $E_{3}, d \mathbf{s}=d \sigma \cdot \mathbf{n}$, in which $d \sigma$ and $\mathbf{n}$ are the area and the unit outward normal vector of a surface element on $S(V)$. 


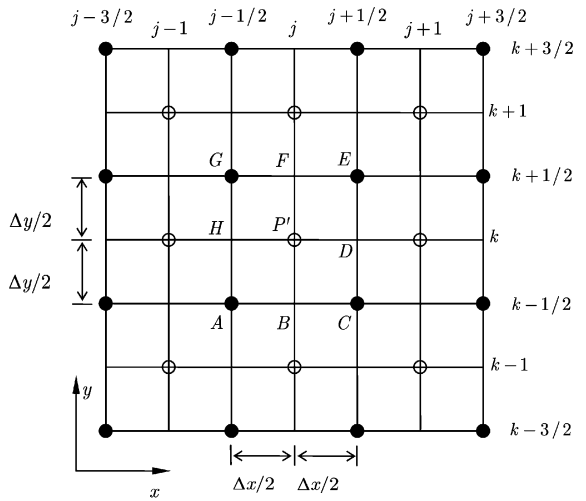

(a) Mesh points projection on the $x-y$ plane

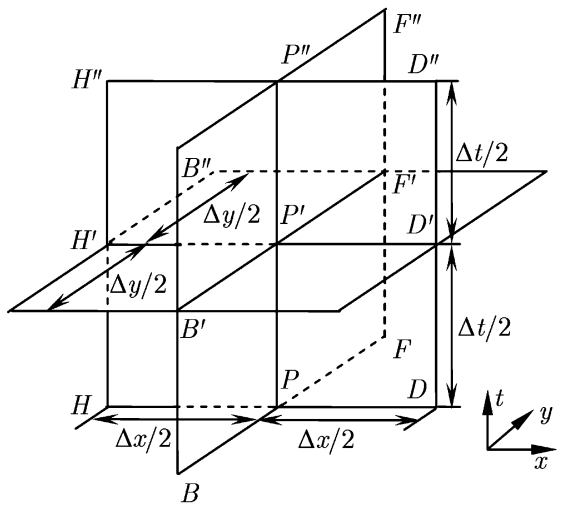

(b) SE

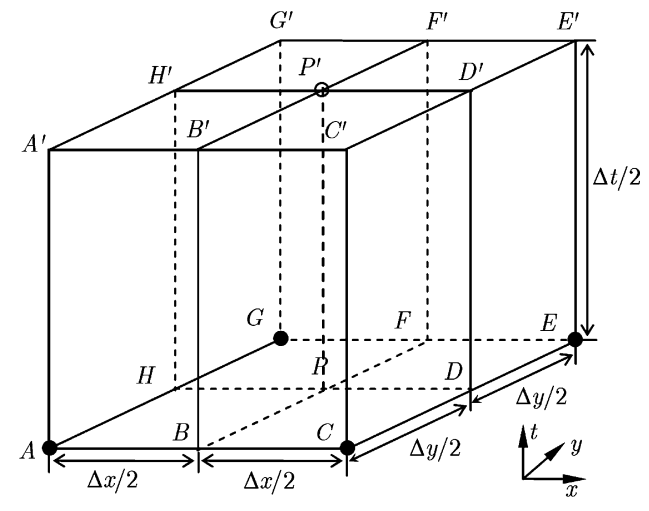

(c) $\mathrm{CE}$

Fig. 2. Space-time geometrical configuration of the improved two-dimensional CE/SE scheme.

For an arbitrary mesh point $P^{\prime}(j, k, n)$ on which the variables are solved, define the solution element $\operatorname{SE}\left(P^{\prime}\right)$ constituted by the three vertical planes intersecting at $P^{\prime}(j, k, n)$ and their neighborhood space as demonstrated in Fig. 2(b). Suppose that $E_{m}, F_{m}$ and $Q_{m}$ at point $(x, y, t)$ in $\operatorname{SE}\left(P^{\prime}\right)$ are approximated by the first-order Taylor's expansions at $P^{\prime}(j, k, n)$, i.e.,

$$
\left\{\begin{array}{l}
Q_{m}(\delta x, \delta y, \delta t)_{P^{\prime}}=\left(Q_{m}\right)_{P^{\prime}}+\left(Q_{m x}\right)_{P^{\prime}} \delta x+\left(Q_{m y}\right)_{P^{\prime}} \delta y+\left(Q_{m t}\right)_{P^{\prime}} \delta t, \\
E_{m}(\delta x, \delta y, \delta t)_{P^{\prime}}=\left(E_{m}\right)_{P^{\prime}}+\left(E_{m x}\right)_{P^{\prime}} \delta x+\left(E_{m y}\right)_{P^{\prime}} \delta y+\left(E_{m t}\right)_{P^{\prime}} \delta t, \\
F_{m}(\delta x, \delta y, \delta t)_{P^{\prime}}=\left(F_{m}\right)_{P^{\prime}}+\left(F_{m x}\right)_{P^{\prime}} \delta x+\left(F_{m y}\right)_{P^{\prime}} \delta y+\left(F_{m t}\right)_{P^{\prime}} \delta t,
\end{array}\right.
$$

where $\delta x=x-x_{P^{\prime}}, \delta y=y-y_{P^{\prime}}, \delta t=t-t_{P^{\prime}} ; x_{P^{\prime}}, y_{P^{\prime}}$ and $t_{P^{\prime}}$ are the position coordinates of point $P^{\prime} .(X)_{P^{\prime}},\left(X_{X}\right)_{P^{\prime}},\left(X_{y}\right)_{P^{\prime}}$ and $\left(X_{t}\right)_{P^{\prime}}$ are the values of $X$ and its first-order derivatives respect to $x, y$ and $t$ at point $P^{\prime}$, respectively, in which $X$ denotes $Q_{m}, E_{m}$ and $F_{m}$, respectively.

Substituting Eq. (21) into Eq. (1), we can obtain

$$
\left(Q_{m t}\right)_{P^{\prime}}=-\left(E_{m x}\right)_{P^{\prime}}-\left(F_{m y}\right)_{P^{\prime}}+\left(S_{m}\right)_{P^{\prime}}
$$

The above equation implies that the variables required in the computation are merely $\left(Q_{m}\right)_{P^{\prime}},\left(Q_{m x}\right)_{P^{\prime}}$ and $\left(Q_{m y}\right)_{P^{\prime}}$, because $E_{m}, F_{m}$ and $S_{m}$ are functions of $Q_{m}$.

Define the conservation element $\operatorname{CE}\left(P^{\prime}\right)$ as demonstrated in Fig. 2(c). From Fig. 2(c), it can be seen that $\operatorname{CE}\left(P^{\prime}\right)$ is related to not only $\operatorname{SE}\left(P^{\prime}\right)$ but also the $\mathrm{SEs}$ of $\operatorname{SE}(A), \operatorname{SE}(C), \operatorname{SE}(E)$ and $\operatorname{SE}(G)$. Note that the values of physical variables on mesh points $A, C, E$ and $G$ are known. Assume that the integral conservation laws are satisfied in every CE. Integrating Eq. (20) on the surfaces of $C E\left(P^{\prime}\right)$ with the aid of Eq. (21), we can obtain

$$
\left(Q_{m}\right)_{P^{\prime}}-\frac{\Delta t}{4}\left(S_{m}\right)_{P^{\prime}}=\frac{1}{4}\left(\bar{Q}+\frac{\Delta t}{\Delta x} \bar{E}+\frac{\Delta t}{\Delta y} \bar{F}+\frac{\Delta t}{4} \bar{S}\right),
$$

where

$$
\left\{\begin{array}{l}
\bar{Q}=Q_{m}\left(\frac{\Delta x}{4}, \frac{\Delta y}{4}, 0\right)_{A}+Q_{m}\left(-\frac{\Delta x}{4}, \frac{\Delta y}{4}, 0\right)_{C}+Q_{m}\left(-\frac{\Delta x}{4},-\frac{\Delta y}{4}, 0\right)_{E}+Q_{m}\left(\frac{\Delta x}{4},-\frac{\Delta y}{4}, 0\right)_{G}, \\
\bar{E}=E_{m}\left(0, \frac{\Delta y}{4}, \frac{\Delta t}{4}\right)_{A}-E_{m}\left(0, \frac{\Delta y}{4}, \frac{\Delta t}{4}\right)_{C}-E_{m}\left(0,-\frac{\Delta y}{4}, \frac{\Delta t}{4}\right)_{E}+E_{m}\left(0,-\frac{\Delta y}{4}, \frac{\Delta t}{4}\right)_{G}, \\
\bar{F}=F_{m}\left(\frac{\Delta x}{4}, 0, \frac{\Delta t}{4}\right)_{A}+F_{m}\left(-\frac{\Delta x}{4}, 0, \frac{\Delta t}{4}\right)_{C}-F_{m}\left(-\frac{\Delta x}{4}, 0, \frac{\Delta t}{4}\right)_{E}-F_{m}\left(\frac{\Delta x}{4}, 0, \frac{\Delta t}{4}\right)_{G} \\
\bar{S}=\left(S_{m}\right)_{A}+\left(S_{m}\right)_{C}+\left(S_{m}\right)_{E}+\left(S_{m}\right)_{G} .
\end{array}\right.
$$

Using the continuous conditions at points $A^{\prime}, C^{\prime}, E^{\prime}$ and $G^{\prime}$, we have the derivatives of $Q_{m}$ with respect to $x$ and $y$

$$
\left(Q_{m x}\right)_{P^{\prime}}=\frac{\left(Q_{m x}\right)_{P^{\prime}}^{+}+\left(Q_{m x}\right)_{P^{\prime}}^{-}}{2}, \quad\left(Q_{m y}\right)_{P^{\prime}}=\frac{\left(Q_{m y}\right)_{P^{\prime}}^{+}+\left(Q_{m y}\right)_{P^{\prime}}^{-}}{2},
$$

where

$$
\left\{\begin{array}{l}
\left(Q_{m x}\right)_{P^{\prime}}^{-}=-\frac{1}{\Delta x}\left[Q_{m}\left(0,0, \frac{\Delta t}{2}\right)_{A}+Q_{m}\left(0,0, \frac{\Delta t}{2}\right)_{G}-2\left(Q_{m}\right)_{P^{\prime}}\right] \\
\left(Q_{m x}\right)_{P^{\prime}}^{+}=+\frac{1}{\Delta x}\left[Q_{m}\left(0,0, \frac{\Delta t}{2}\right)_{C}+Q_{m}\left(0,0, \frac{\Delta t}{2}\right)_{E}-2\left(Q_{m}\right)_{P^{\prime}}\right] \\
\left(Q_{m y}\right)_{P^{\prime}}^{-}=-\frac{1}{\Delta y}\left[Q_{m}\left(0,0, \frac{\Delta t}{2}\right)_{A}+Q_{m}\left(0,0, \frac{\Delta t}{2}\right)_{C}-2\left(Q_{m}\right)_{P^{\prime}}\right] \\
\left(Q_{m y}\right)_{P^{\prime}}^{+}=+\frac{1}{\Delta y}\left[Q_{m}\left(0,0, \frac{\Delta t}{2}\right)_{E}+Q_{m}\left(0,0, \frac{\Delta t}{2}\right)_{G}-2\left(Q_{m}\right)_{P^{\prime}}\right] .
\end{array}\right.
$$




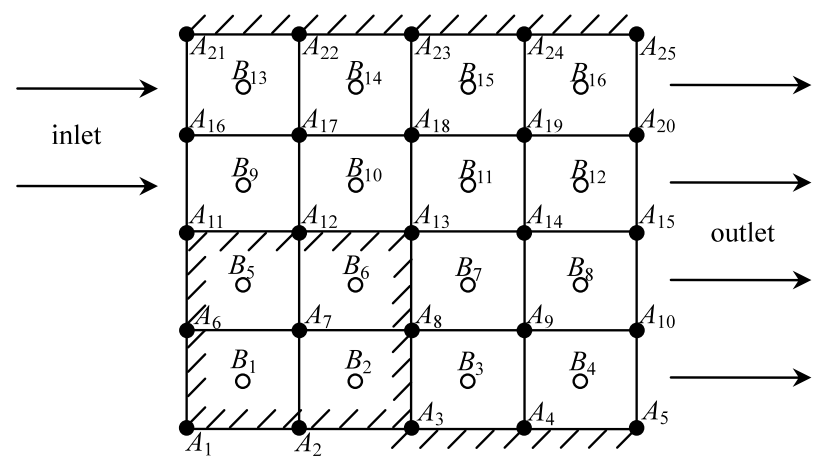

Fig. 3. Sketch map of computational grid.

To avoid numerical instability in discontinuous cases, replace Eq. (25) by

$$
\left(Q_{m x}\right)_{P^{\prime}}=W\left[\left(Q_{m x}\right)_{P^{\prime}}^{-},\left(Q_{m x}\right)_{P^{\prime}}^{+}, \alpha\right], \quad\left(Q_{m y}\right)_{P^{\prime}}=W\left[\left(Q_{m y}\right)_{P^{\prime}}^{-},\left(Q_{m y}\right)_{P^{\prime}}^{+}, \alpha\right],
$$

where $\alpha$ is an adjustable constant and usually equals $1-2$, and the weighted equation is

$$
W\left[x_{-}, x_{+}, \alpha\right]=\frac{\left|x_{+}\right|^{\alpha} x_{-}+\left|x_{-}\right|^{\alpha} x_{+}}{\left|x_{+}\right|^{\alpha}+\left|x_{-}\right|^{\alpha}} .
$$

Obviously, Eq. (27) is same as Eq. (25) when $\alpha=0$. In this work, $\alpha$ is equal to 1.

It should be noted that $\left(Q_{m}\right)_{P^{\prime}}$ cannot be obtained explicitly from Eq. (23) due to the source term $\left(S_{m}\right)_{P^{\prime}}$. As $\left(S_{m}\right)_{P^{\prime}}$ is a function of $\left(Q_{m}\right)_{P^{\prime}}$, a local Newton iterative procedure is usually needed to determine $\left(Q_{m}\right)_{P^{\prime}}$. In the present work, to avoid the iterative procedure and save computation time, we replace $\left(S_{m}\right)_{P^{\prime}}$ by their linear prediction of current time in Eq. (23), i.e.,

$$
\left\{\begin{array}{l}
\left(Q_{m}\right)_{P^{\prime}}=\frac{1}{4} \bar{Q}+\frac{\Delta t}{4 \Delta x} \bar{E}+\frac{\Delta t}{4 \Delta y} \bar{F}+\frac{\Delta t}{2} \tilde{S}, \\
\tilde{S}=\bar{S}+\frac{\Delta t}{2} \bar{S}_{t},
\end{array}\right.
$$

where $\bar{S}_{t}$ is the time derivative of $\bar{S}$. $\left(Q_{m}\right)_{P^{\prime}}$ can be solved directly without any iteration, since the time derivatives of $\left(S_{m}\right)_{A}$, $\left(S_{m}\right)_{C}$, $\left(S_{m}\right)_{E}$ and $\left(S_{m}\right)_{G}$ are all known at current time. In our numerical tests, the results of linear approximation agree with those of Newton iteration, which indicates that the linear approximation of $\left(S_{m}\right)_{P^{\prime}}$ is reasonable.

\subsection{Boundary conditions}

Fig. 3 shows the sketch map of computational grid for shock wave diffraction at a right angle edge. At an initial time, we should assign physical quantities and their first-order derivatives at grid point $A_{i}(i=3-5,8-10,11-25)$ if the improved CE/SE scheme is adopted to solve this problem. Physical quantities and their first-order derivatives at $B_{j}(j=3,4,7-16)$ can been calculated at the first half time step, and physical quantities and their first-order derivatives at $A_{i}(i=9,14,17-19)$ can be calculated when a time step over. So the physical quantities and their first-order derivatives at $A_{i}(i=3-5,8,10-13,15,16,20-25)$ should be assigned based on each special boundary conditions.

\section{(a) Solid boundary/wall boundary}

The solid boundary is the most common boundary encountered in CFD. We consider a solid wall " $A_{21} A_{25}$ ", which is parallel to the $x$-direction. The physical quantities and their first-order derivatives at point $A_{23}$ can been gained by

$$
\left\{\begin{array}{l}
\xi_{A_{23}}=\xi_{A_{18}}, \quad \xi_{x, A_{23}}=\xi_{x, A_{18}}, \quad \xi_{y, A_{23}}=\xi_{y, A_{18},}, \\
u_{A_{23}}=u_{A_{18}}, \quad u_{x, A_{23}}=u_{x, A_{18}}, \quad u_{y, A_{23}}=0, \\
v_{A_{23}}=0, \quad v_{x, A_{23}}=0, \quad v_{y, A_{23}}=v_{y, A_{18}},
\end{array}\right.
$$

where $\xi$ denotes $\rho_{g}, \rho_{i}, p, \alpha, \beta, \rho_{p}, N ; u$ denotes $u_{g}$ and $u_{p} ; v$ denotes $v_{g}$ and $v_{p}$.

For the corner point, such as point $A_{13}$, i.e. the straight line " $A_{3} A_{13}$ " and " $A_{11} A_{13}$ " are solid walls. The physical quantities and their first-order derivatives at point $A_{13}$ can been gained by

$$
\left\{\begin{array}{l}
\xi_{A_{13}}=\xi_{A_{19}}, \quad \xi_{x, A_{13}}=\xi_{x, A_{19}}, \quad \xi_{y, A_{13}}=\xi_{y, A_{19}}, \\
u_{A_{13}}=0, \quad u_{x, A_{13}}=u_{x, A_{14}}, \quad u_{y, A_{13}}=0 \\
v_{A_{13}}=0, \quad v_{x, A_{13}}=0, \quad v_{y, A_{13}}=v_{y, A_{18}} .
\end{array}\right.
$$

\section{(b) Inlet/outlet boundary}

The inlet and outlet boundaries are also the most common boundaries. Suppose the straight line " $A_{11} A_{21}$ " is an inlet boundary, and the flow direction is uniformly from the left to the right (see Fig. 3 ). The physical quantities and their first-order derivatives at point $A_{16}$ can been gained by 


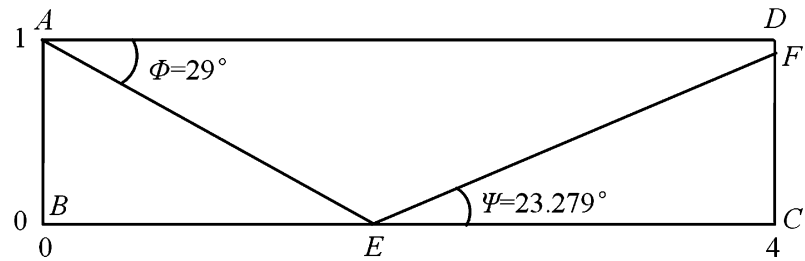

Fig. 4. Structural scheme of the shock reflection problem.

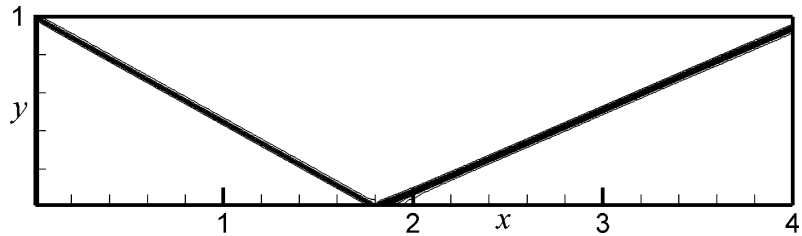

Fig. 5. Density contour by improved CE/SE scheme $(400 \times 100)$.

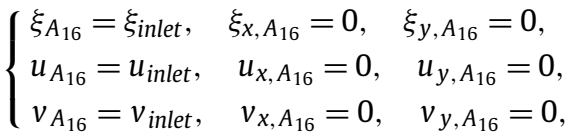

where physical quantities with subscript "inlet" are the reference values of the inlet.

For the outlet boundary " $A_{5} A_{25}$ ", we suppose there is no back flow and the outside flow field does not affect the computational flow field. The physical quantities and their first-order derivatives at point $A_{15}$ can been gained by

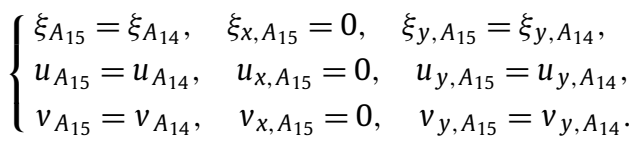

\section{Applications}

\subsection{Shock wave reflection}

Shock wave reflection is a classical benchmark problem in CFD [24]. An oblique incident and a reflected shock wave can be generated above a flat plate by imposing suitable upstream conditions. Fig. 4 shows the structural scheme and the exact solution of this problem. The domain is a $4 \times 1$ rectangle. The left boundary conditions are $\rho_{\text {inlet }}=1.0, u_{\text {inlet }}=2.9, v_{\text {inlet }}=0, p_{\text {inlet }}=0.7143$; the upper boundary conditions are $\rho_{\text {inlet }}=1.7, u_{\text {inlet }}=2.6193, v_{\text {inlet }}=-0.50632, p_{\text {inlet }}=1.5282$ (the variables used in this case are dimensionless [24]). The right boundary is free (outlet boundary); and the bottom boundary is solid wall. The total computing time is 2.5 with CFL number equals to 0.4 .

Fig. 5 shows the density contour computed by the improved CE/SE scheme on $400 \times 100$ uniform meshes. The numerical shock wave location agrees well with the exact solution (Fig. 4). In order to evaluate the accuracy of the improved CE/SE scheme further, we also calculated the shock reflection problem under the same conditions using the first-order Roe scheme and the second-order MUSCL scheme. Fig. 6(a) shows the distributions of the pressure coefficient $C_{p}\left(=2\left(p / p_{\infty}-1\right) /\left(\gamma M_{\infty}^{2}\right)\right)$ at $y=0.5$ using different numerical schemes, and the exact solution is also provided. Fig. 6(b) gives a local enlargement vision of Fig. 6(a). From Fig. 6 we can find that the accuracy of the improved CE/SE scheme is higher than the first-order Roe scheme and the second-order MUSCL scheme on the same $400 \times 100$ meshes. Fig. 6 also shows numerical results using the improved CE/SE scheme with $200 \times 50$ meshes and $800 \times 200$ meshes, respectively. The numerical results converge to the exact solution as the number of computing meshes increases.

\subsection{Shock wave diffraction in gas-particle mixtures}

Shock wave propagation and diffraction phenomena in gas-particle mixtures were the subject of numerous experimental, theoretical and numerical studies in recent decades. In this paper, the problem considers a flat duct with an abrupt expansion of the cross section, filled with air-sand mixture. The governing equations for the air-sand two-phase flow are Eq. (1) and Eq. (2). The computation model is shown in Fig. 7. The length of " $A B$ " is $0.8 \mathrm{~m}$, "BC" $0.4 \mathrm{~m}$, " $C D$ " $0.4 \mathrm{~m}$, " $D E$ " $0.2 \mathrm{~m}$. The computational mesh number is $800 \times 400$. The boundary of " $A F$ " is inlet, the others are solid boundaries. The velocities of gas phase and particle phase are zero at the initial time $\left(u_{g}=0\right.$, $v_{g}=0, u_{p}=0, v_{p}=0$ ). The initial density, temperature and pressure of the gas phase are $1.186 \mathrm{~kg} / \mathrm{m}^{3}, 298 \mathrm{~K}$ and $1.01325 \times 10^{5} \mathrm{~Pa}$, respectively. A shock wave with Mach number $M a=3.0$ is generated at " $A F$ " in the initial time. The inlet physical quantities of gas phase can be gained based on the shock relations. The density of particle phase, $\rho_{p}$, is $0.5 \mathrm{~kg} / \mathrm{m}^{3}$, and the particle radius, $r$, is $50 \mu \mathrm{m}$. The density of sand, $\rho_{\text {sand }}$, is $2000 \mathrm{~kg} / \mathrm{m}^{3}$. The particle number per unit volume, $N$, can be gained by Eq. (4).

Fig. 8 shows the pressure contours (Fig. 8(a)), density field of gas phase (Fig. 8(b)) and density field of particle phase (Fig. 8(c)) at $0.70 \mathrm{~ms}$. The leading shock wave includes three parts, incident shock (IS), diffracted shock (DS), and reflected shock with Mach stem (MS). An expansion fan (EF) is formed in the neighborhood of the backward-facing step. The flow behind the backward-facing step is characterized by the presence of a vortex (V), secondary shock (SS), contact surface (CS, see the dashed line in Fig. 8(a) or see Fig. 8(b)), and attached shock (AS). In order to compare the numerical results, a shock wave diffraction $(M a=3)$ in gas is also simulated by solving the two-dimensional Euler equation using the improved CE/SE scheme. Fig. 9 can agree well with corresponding research [25,26] and shows 


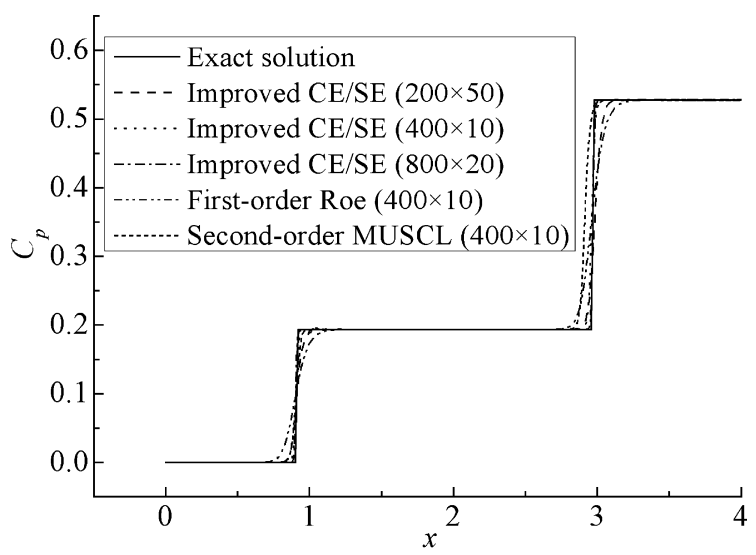

(a) The whole $x$ axis

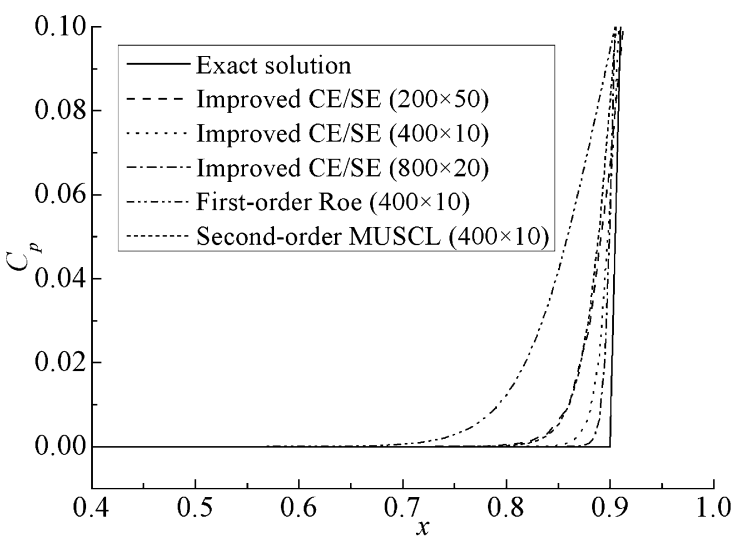

(b) Local enlargement

Fig. 6. Pressure coefficients at $y=0.5$ by different numerical schemes and computational meshes.

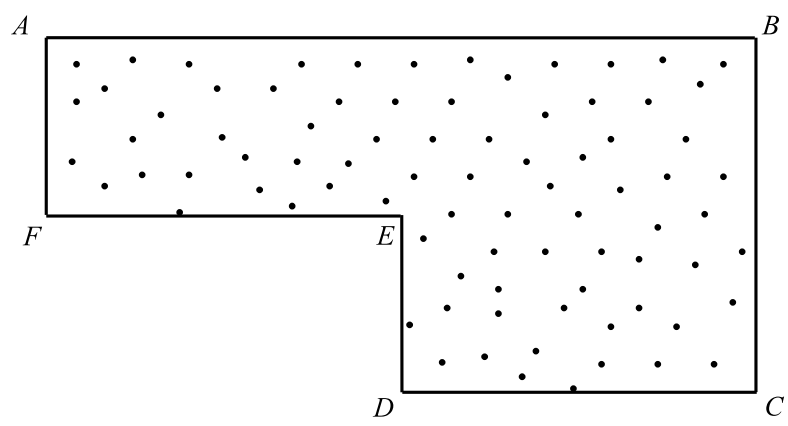

Fig. 7. The schematic computation model.

the accuracy of the improved CE/SE schemes. Comparing Fig. 8 with Fig. 9, we can find that typical features of shock wave diffraction in a dilute gas-particle mixture are similar to shock wave diffraction in gases, and Fedorov et al. reached the same conclusion [27].

From Fig. 8(c) we can find that the maximum density of particle phase is appear near the secondary shock (SS). And the change of particle phase density near the shock wave front is smooth, which is different with the density of gas phase (see Fig. 8(b)). Fig. 10 shows the particle number, gas phase velocity and particle phase velocity at $y=0.3 \mathrm{~m}$. We can find that the velocity of gas phase increases immediately as the shock wave passes (which is similar to shock wave propagating in gas). However, the velocity of particle phase increases slowly and achieves the maximum value at $x=0.414 \mathrm{~m}$. The reason is that the density of sand is much bigger than the density of gas, so the force acting on the sand particles cannot accelerate the sand particle immediately. From Fig. 10, we also can find that the particle number achieves the maximum value at the region near $x=0.42 \mathrm{~m}$, and then decreases almost to zero. The reason is that there is no particle supplying to the flow field (the boundary "AF" is just an inlet for gas phase).

\subsection{Synthesis of $\mathrm{TiO}_{2}$ nanoparticle}

In this section, detonation synthesis of nanosized titania $\left(\mathrm{TiO}_{2}\right)$ particle is simulated. The materials for the synthesis are $\mathrm{TiCl}_{4}, \mathrm{O}_{2}$ and $\mathrm{H}_{2}$. The chemical reaction in the reaction vessel can be described as

$\mathrm{TiCl}_{4}$ (gas) $+\mathrm{O}_{2}$ (gas) $+2 \mathrm{H}_{2}$ (gas) $\rightarrow \mathrm{TiO}_{2}$ (solid) $+4 \mathrm{HCl}$ (gas) 


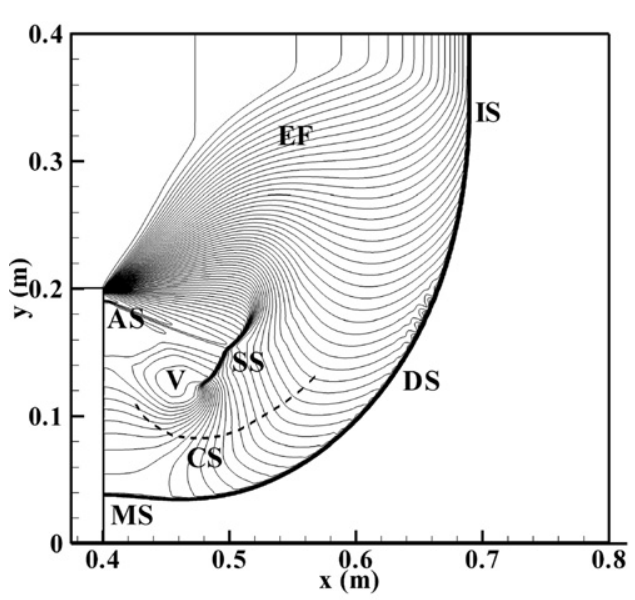

(a) Pressure contours

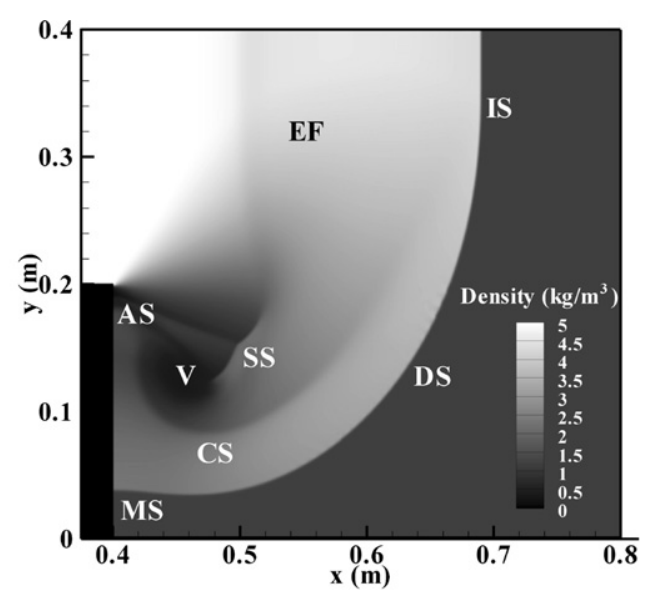

(b) Density field of gas phase

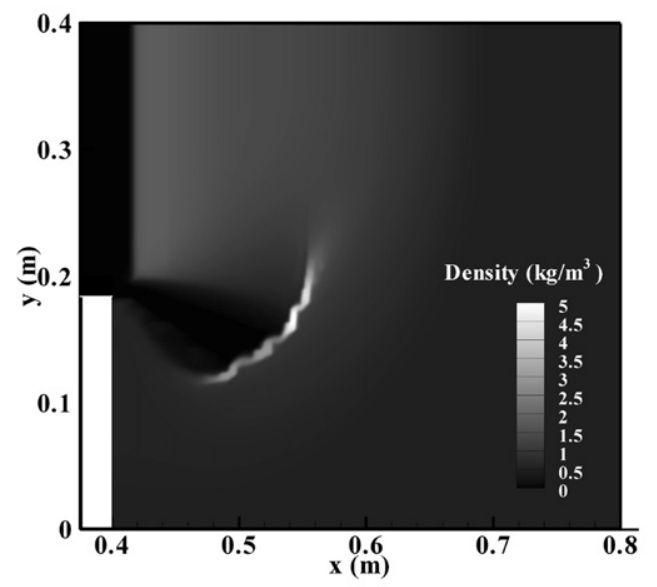

(c) Density field of particle phase

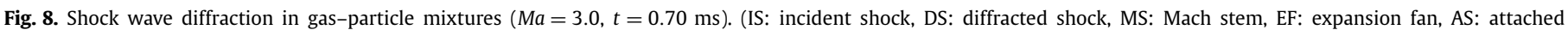
shock, SS: secondary shock, V: vortex, CS: contact surface.)

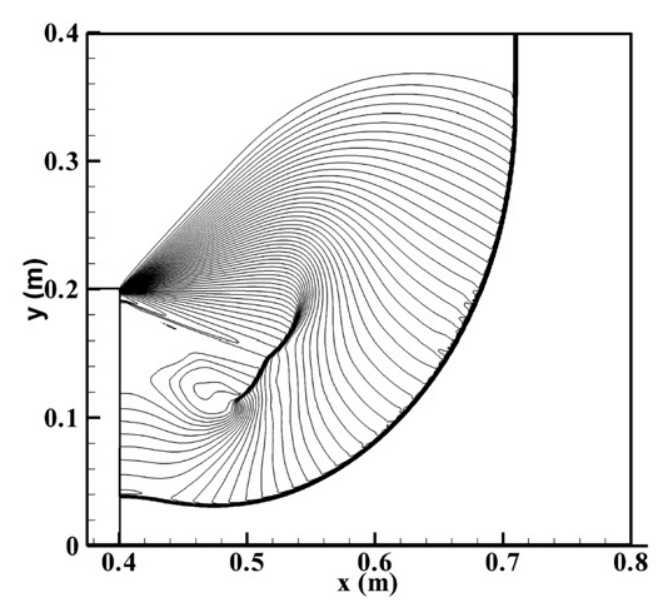

(a) Pressure contours

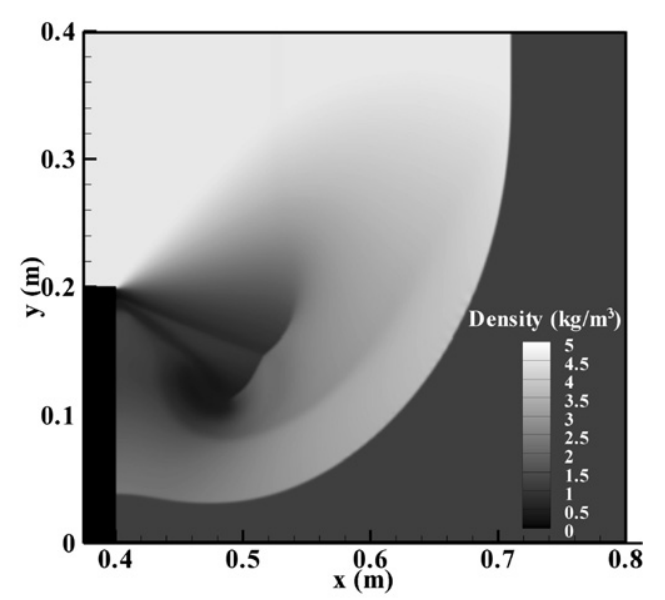

(b) Gas density field

Fig. 9. Shock wave diffraction in gas $(M a=3.0, t=0.68 \mathrm{~ms})$.

The rate of particle growth $I_{p}$ is coupled with the chemical reaction model [20]

$$
I_{p}=N k_{0} \exp \left(-\frac{E_{a}}{R T}\right) c\left(\mathrm{TiCl}_{4}\right),
$$

where the constant rate $k_{0}=8.29 \times 10^{4} \mathrm{~s}^{-1}, E_{a}=88.8 \mathrm{~kJ} / \mathrm{mol}, c\left(\mathrm{TiCl}_{4}\right)$ is the molarity of $\mathrm{TiCl}_{4}$.

The computation model is a closed channel (length $50 \mathrm{~mm}$, width $50 \mu \mathrm{m}$ ). The number of computational grids is $2000 \times 2$. So this computation can be seen as a one-dimensional situation. The molarity ratio of the three gaseous materials is $c\left(\mathrm{TiCl}_{4}\right): c\left(\mathrm{O}_{2}\right): c\left(\mathrm{H}_{2}\right)=1: 1: 2$ at the initial time. The initial density, pressure and temperature are $2.0 \mathrm{~kg} / \mathrm{m}^{3}, 1.01325 \times 10^{5} \mathrm{~Pa}$ and $343 \mathrm{~K}$, respectively. In order to 


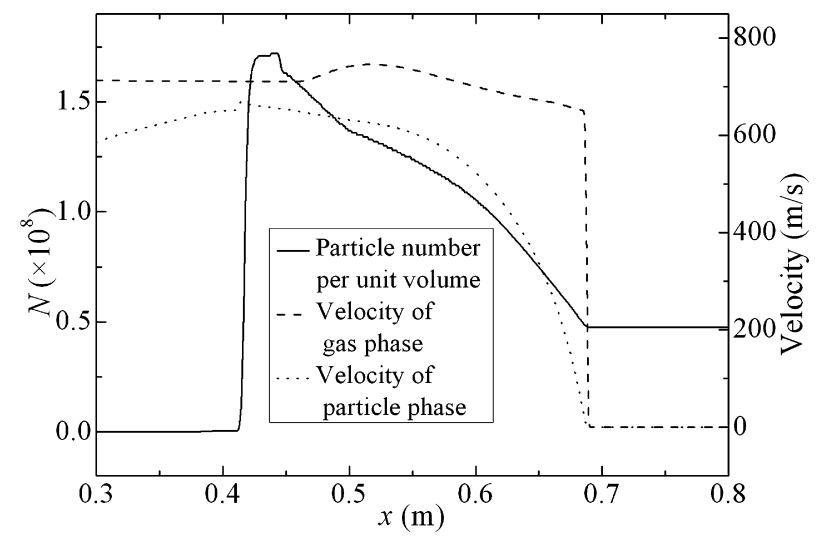

Fig. 10. Particle number and the velocities profiles at $y=0.3 \mathrm{~m}$.

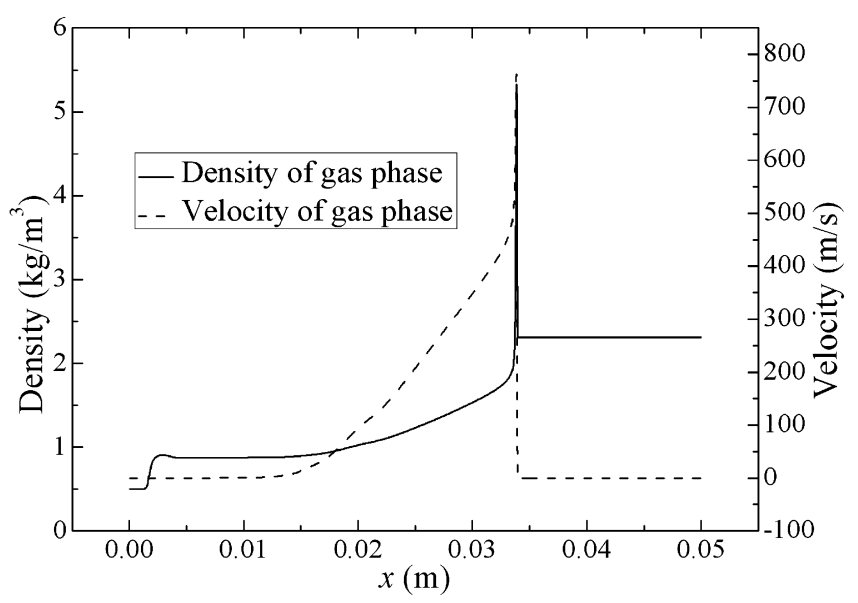

(a) Density and velocity of gas phase profiles

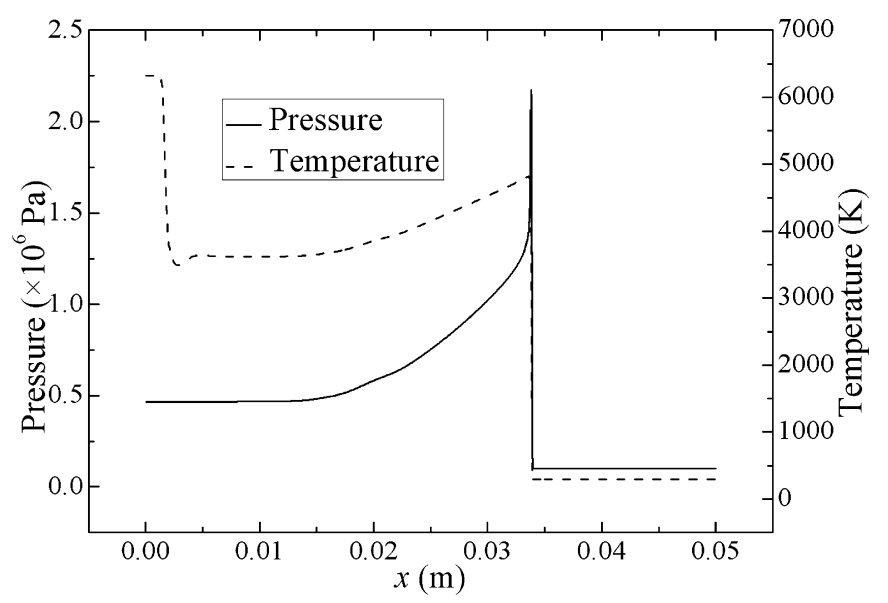

(b) Pressure and temperature profiles

Fig. 11. Profile of physical quantities at $t=3.0 \times 10^{-5} \mathrm{~s}$.

form a detonation wave, there is a high initial pressure and temperature $\left(2.0265 \times 10^{6} \mathrm{~Pa}\right.$ and $6860 \mathrm{~K}$, respectively $)$ region in the channel left $(1 \mathrm{~mm})$. The other computing parameters are given as below: $\rho_{\text {titania }}=4300 \mathrm{~kg} / \mathrm{m}^{3}, Q=6.5 \times 10^{5} \mathrm{~J} / \mathrm{kg}, \mu_{\mathrm{g}}=2.07 \times 10^{-5} \mathrm{Pas}$, $\mu_{p}=3.5 \times 10^{-4}$ Pa s.

Fig. 11 shows the gas density, velocity, pressure and temperature at $30 \mu \mathrm{s}$. The profiles of gas velocity (see Fig. 11(a)), pressure and temperature (see Fig. 11(b)) are very similar to the structure of a gaseous detonation wave. But the profile of gas density (see Fig. 11(a)) has a sudden reduce due to the phase transition from gas to solid particle. Fig. 12 shows the local enlargement profiles of the particle radius and the progress parameter of chemical reaction. The process of detonation synthesis can been observed from Figs. 11 and 12 . The particle radius increases rapidly near the detonation front where the maximum pressure and temperature appear (see Fig. 11). Further more, the rate of particle growth is closely related to the rate of chemical reaction (see Fig. 12).

The particle radius in our simulation is about $29.3 \mathrm{~nm}$ and keeps this size behind the C-J point. In the same experiment condition, the $\mathrm{TiO}_{2}$ sample includes octahedrite (68.37\%) and titanic schorl (31.63\%). And the average grain size of anatase and rutile calculated by XRD 




Fig. 12. Local enlargement profiles of the particle radius and the progress parameters of chemical reactions.

using the Scherrer equation [28] is between 20 and $40 \mathrm{~nm}$ [29]. The peak pressure in our simulation is $2.17 \mathrm{MPa}$, which also can agree well with the experimental data $2.03 \mathrm{MPa}$ [29] (the relative error is 5.85\%).

\subsection{Propagation of gas-droplet two-phase detonation}

In this section, two-phase planar detonations in dilute oxygen-droplet system are simulated. The fuel droplets are made of kerosene and with a uniform radius. Kerosene is an extremely complex mixture with many components and their exact composition and properties vary from batch to batch. Wang had proposed a one-formula surrogate fuel $\left(\mathrm{C}_{12} \mathrm{H}_{24}\right)$ formulation and corresponding chemical kinetics models were given [30]. The detail of the reduced reaction mechanism can be found in Ref. [30].

The channel walls are still considered as solid boundaries (length $0.2 \mathrm{~m}$, width $0.2 \mathrm{~mm}$ ). The number of computational grids is $2000 \times 2$. At initial time, the gas density, pressure and temperature are $1.3 \mathrm{~kg} / \mathrm{m}^{3}, 1.01325 \times 10^{5} \mathrm{~Pa}$ and $298 \mathrm{~K}$, respectively. The density of kerosene, $\rho_{\text {fuel }}$, equals to $800 \mathrm{~kg} / \mathrm{m}^{3}$. The radii of the droplets, $r$, equals to $10 \mu \mathrm{m}$. The equivalent ratio of the $\mathrm{O}_{2}-\mathrm{C}_{12} \mathrm{H}_{24}$ system is equal to 1.0 . The density of particle phase equals to $0.382 \mathrm{~kg} / \mathrm{m}^{3}$ and the droplet number per unit volume equals to $9.11 \times 10^{8}$. The other computing parameters of the stoichiometric $\mathrm{O}_{2}-\mathrm{C}_{12} \mathrm{H}_{24}$ system are given as below: $\lambda=0.10 \mathrm{~W} \mathrm{~K}^{-1} \mathrm{~m}^{-1}, L=5.27 \times 10^{5} \mathrm{~J} / \mathrm{kg}, \mu_{\mathrm{g}}=2.07 \times 10^{-5} \mathrm{~Pa}$, $\mu_{p}=3.50 \times 10^{-4} \mathrm{Pas}, \operatorname{Pr}=0.74$. Detonation wave is generated by igniting in the left $(1 \mathrm{~mm})$ with a high initial pressure and temperature as $1.01325 \times 10^{6} \mathrm{~Pa}$ and $2980 \mathrm{~K}$, respectively.

Fig. 13 shows the distributions of the density of gas phase, the velocity of gas phase, the pressure and the temperature in $x$ direction at different time. It can be seen that the DDT (Deflagration-to-Detonation Transition) process completes at about $20 \mu \mathrm{s}$, which is much longer than DDT time of gas case. For the similar ignition condition, the DDT process in stoichiometric $\mathrm{H}_{2}-\mathrm{O}_{2}$ mixture completes less than $1 \mu \mathrm{s}$ in our simulations. The profiles of the density, velocity and are similar to those of gaseous detonations, but values of physical quantities are higher than those of gaseous detonations. That is because the kerosene has higher energy density than hydrogen. Fig. 13(d) shows that the peak temperature is behind the detonation front. That is because the separation and evaporation of kerosene droplets take a considerable time and the chemical reaction rate of hydrocarbon fuel is less than hydrogen.

In order to verify the accuracy of the improved CE/SE scheme in two-phase detonations, we also simulated two-phase planar detonations in $\mathrm{O}_{2}-\mathrm{C}_{6} \mathrm{H}_{14}$ system with different equivalence ratios $(\varphi=0.3,0.35,0.41,0.49,0.56$ and 0.68$)$. Fig. 14 shows detonation velocities at different equivalence ratios with corresponding results from C-J theory and experiments [31,32]. From Fig. 14 we can find that the numerical results and C-J theory results have the same trend, but the experiment results have an obvious error near $\varphi=0.5$. The experimental errors in two-phase detonations are much larger than that in gaseous detonations, because the experimental conditions of two-phase detonations, such as the sizes and distribution of droplets, are quite difficult to be well-proportioned. But people prefer to trust in experimental results because errors of C-J theory which ignores two-phase effects are larger [32]. The relative errors of simulations and C-J theory are also shown in Fig. 14 (see the dash lines). The relative errors of C-J theory are greater than $13.5 \%$ in all the six cases, and the maximum relative error is $22.9 \%$. The relative errors of simulations range from $-16.8 \%$ to $8.9 \%$, especially the numerical results can agree well with experiment date at $\phi=0.5-0.6$. So we think the numerical simulations are more accurate than the C-J theory for the two-phase planar detonation problems.

\section{Conclusion}

In this paper, the improved CE/SE scheme are developed for dilute gas-particle flows. This scheme is based on the rectangular mesh and can be constructed easily. The two-fluid model is used to describe the gas-particle two-phase system, and two chemical reaction models and their governing equations were introduced for detonation synthesis and two-phase detonation propagation. Obviously, other chemical and physical models can be added to the current frame conveniently.

The main advantages of the improved CE/SE scheme are clear in physical concept, easy to be extended to three-dimensional situations, accurate for capturing shock wave and convenient to implement boundary conditions. In the applications to dilute gas-particle flow simulations, this scheme combines chemical reaction models with physics models conveniently and treats source items easily. The numerical results show that the improved CE/SE scheme can simulate dilute gas-particle flows accurately. Thus, the improved CE/SE scheme can be applied to dilute gas-particle flow simulations for academic explore and engineering extensively. 


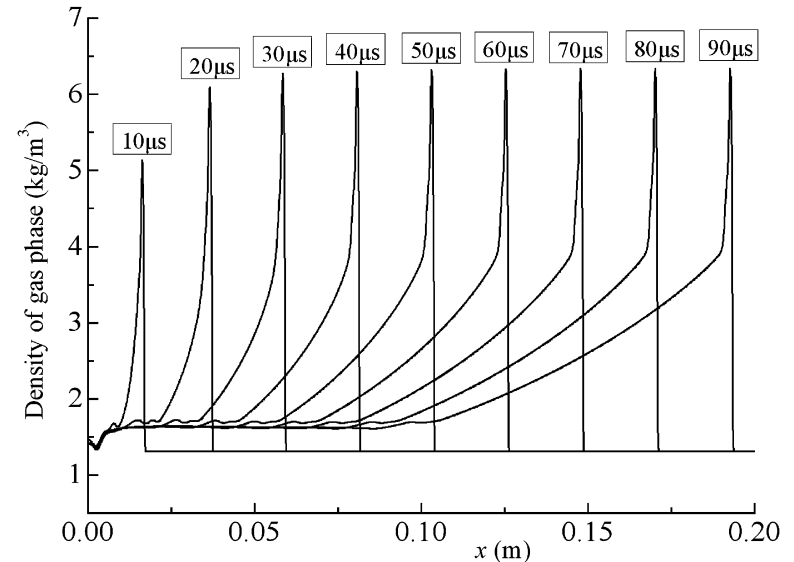

(a) The density of gas phase

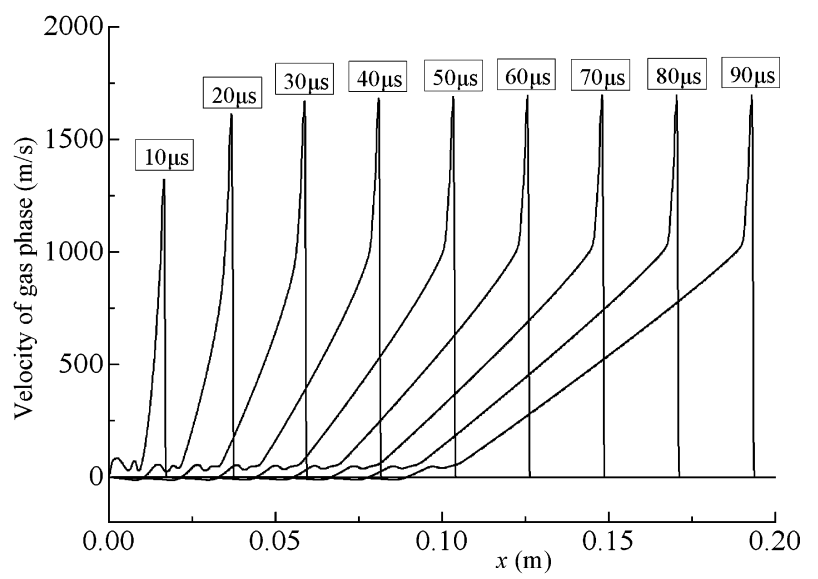

(b) The velocity of gas phase

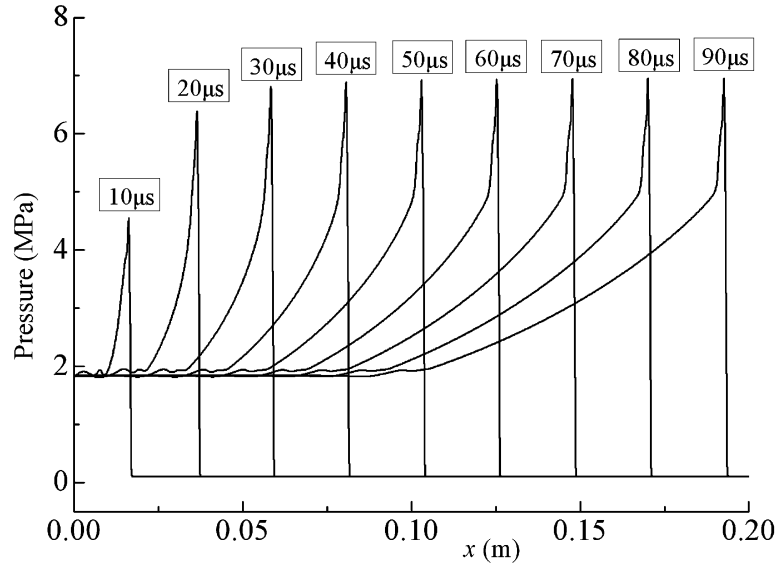

(c) The pressure

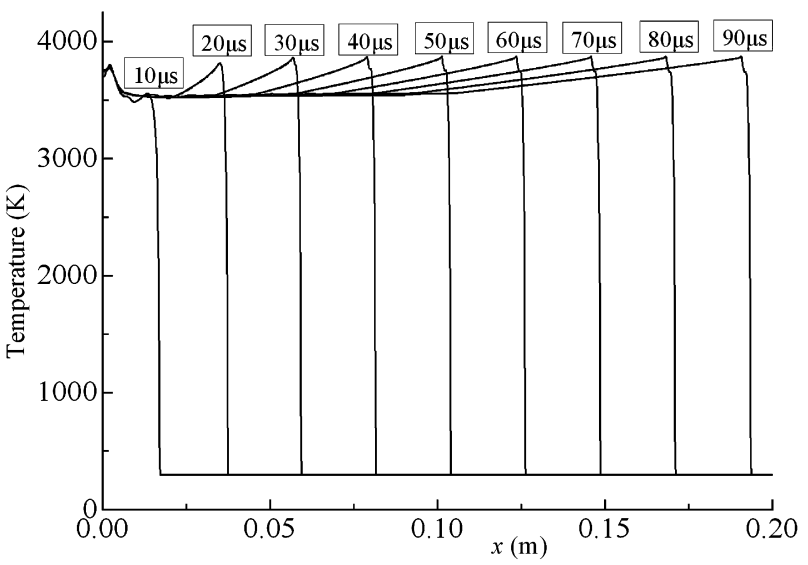

(d) The temperature

Fig. 13. Physical quantity profiles of $\mathrm{O}_{2}-\mathrm{C}_{3} \mathrm{H}_{6} \mathrm{O}$ two-phase detonation wave at $t=1 \times 10^{-3} \mathrm{~s}$ (4000 meshes).

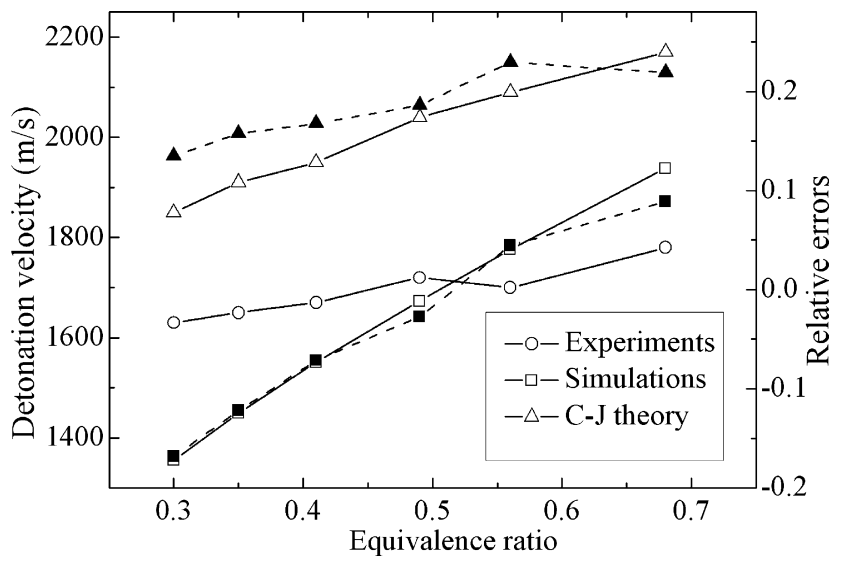

Fig. 14. $\mathrm{O}_{2}-\mathrm{C}_{6} \mathrm{H}_{14}$ two-phase detonation velocities at different equivalence ratios (solid line for detonation velocity, dash line for relative error).

\section{References}

[1] R.O. Fox, A quadrature-based third-order moment method for dilute gas-particle flows, Journal of Computational Physics 227 (12) (2008) $6313-6350$.

[2] S. Yuu, T. Ueno, T. Umekage, Numerical simulation of the high Reynolds number slit nozzle gas-particle jet using subgrid-scale coupling large eddy simulation, Chemical Engineering Science 56 (14) (2001) 4293-4307.

[3] S.C. Chang, The method of space-time conservation element and solution element - a new approach for solving the Navier-Stokes and Euler equations, Journal of Computational Physics 119 (2) (1995) 295-324.

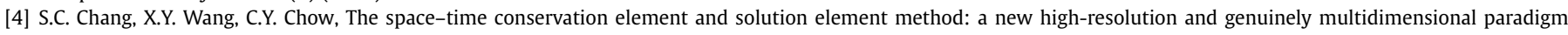
for solving conservation laws, Journal of Computational Physics 156 (1999) 89-136.

[5] X.Y. Wang, C.Y. Chow, S.C. Chang, The space-time conservation element and solution element method - a new high-resolution and genuinely multidimensional paradigm for solving conservation laws II. Numerical simulation of shock waves and contact discontinuities, NASA/TM 1998-208844, 1998.

[6] Z.C. Zhang, S.T. John Yu, S.C. Chang, A space-time conservation element and solution element method for solving the two- and three-dimensional unsteady Euler equations using quadrilateral and hexahedral meshes, Journal of Computational Physics 175 (2002) 168-199.

[7] Y.H. Guo, A.T. Hsu, J. Wu, Z.G. Yang, A. Oyediran, Extension of CE/SE method to 2D viscous flows, Computers \& Fluids 33 (2004) $1349-1361$. 


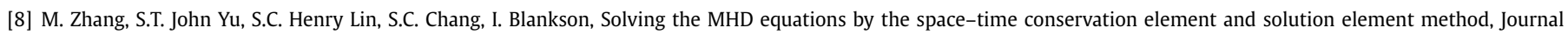
of Computational Physics 214 (2) (2006) 599-617.


587-606.

[10] J.T. Wang, K.X. Liu, D.L. Zhang, An improved CE/SE scheme for multi-material elastic-plastic flows and its applications, Computers and Fluids 38 (2009) 544-551.

[11] Z.C. Zhang, S.T. John Yu, H. Hao, S.C. Chang, Direct calculations of two- and three-dimensional detonations by an extended CE/SE method, AIAA 2001-0476, 2001.

[12] H. Hao, S.T. John Yu, Z.C. Zhang, Direct calculations of one-, two-, and three-dimensional detonations by the CESE method, AIAA $2005-0229,2005$.

[13] L.B. David, S.T. John Yu, Simulation of shock-tube flows with detailed finite-rate chemistry by the CESE method, AIAA 2009-0811, 2009.

[14] K.X. Liu, J.T. Wang, Analysis of high accuracy conservation-element and solution-element schemes, Chinese Physics Letters 21 (11) (2004) $2085-2088$.

[15] G. Wang, D.L. Zhang, K.X. Liu, An improved CE/SE scheme and its application to detonation propagation, Chinese Physics Letters 24 (12) (2007) $3563-3566$.

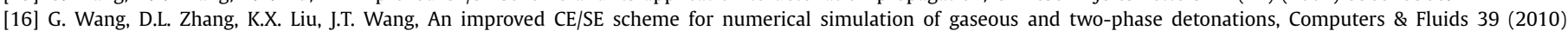
$168-177$.

[17] A.A. Ranger, J.A. Nicholls, Aerodynamic shattering of liquid drops, AIAA Journal 7 (2) (1969) 285-290.

[18] S. Eidelman, A. Burcat, Evolution of a detonation wave in a cloud of fuel droplet: Part I. Influence of igniting explosion, AIAA Journal 18 (9) (1980) 1103-1109.

[19] S. Taki, T. Fujiwara, Numerical simulation on the establishment of gaseous detonation, Progress in Astronautics and Aeronautics 94 (1984) $186-200$.

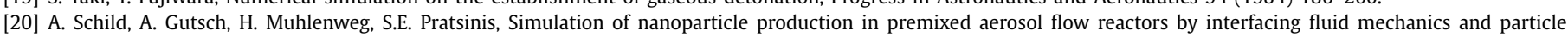
dynamics, Journal of Nanoparticle Research 1 (1999) 305-315.

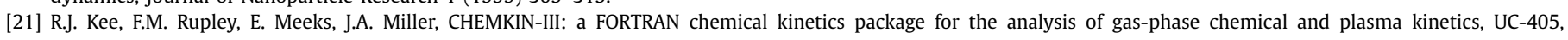
SAND96-8216, 1996.

[22] B.J. McBride, M.J. Zehe, S. Gordon, NASA Glenn coefficients for calculating thermodynamic properties of individual species, NASA/TP $2002-211556,2002$.

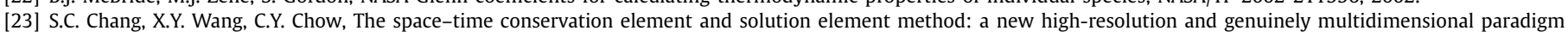
for solving conservation laws, Journal of Computational Physics 156 (1999) 89-136.

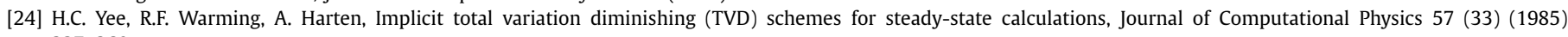
327-360.

[25] B.W. Skews, The perturbed region behind a diffracting shock wave, Journal of Fluid Mechanics 29 (4) (1967) 705-719.

[26] R. Hillier, Computation of shock wave diffraction at a ninety degrees convex edge, Shock Waves 1 (2) (1991) 89-98.

[27] A.V. Fedorov, T.A. Khmel, Y.V. Kratova, Shock and detonation wave diffraction at a sudden expansion in gas-particle mixtures, Shock Waves 18 (4) (2008) $281-290$.

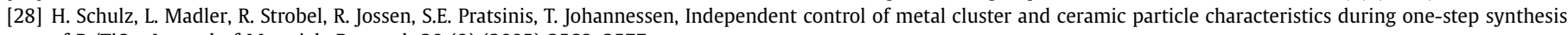
of Pt/TiO

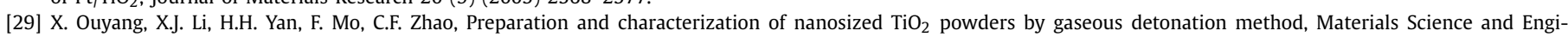
neering B - Advanced Functional Solid-State Materials 153 (1-3) (2008) 21-24.

[30] T.S. Wang, Thermophysics characterization of kerosene combustion, AIAA 2000-2511, 2000.

[31] E.K. Dabora, K.W. Ragland, J.A. Nicholls, A study of heterogeneous detonations, Astronautica Acta 12 (1966) 9-16.

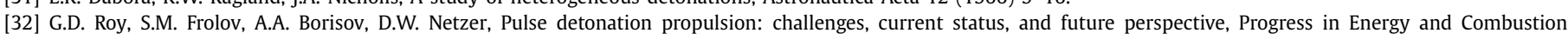
Science 30 (2004) 545-672. 